\title{
Rapid response to biomedical challenges and threats
}

\section{Wladek Minor ${ }^{1}$, Mariusz Jaskolski², Alexander Wlodawer ${ }^{3}$, Zbigniew Dauter $^{3}$, Joanna Macnar ${ }^{4}$, Dariusz Brzezinski ${ }^{1}$, David Cooper ${ }^{1}$, Marcin Kowiel ${ }^{7}$, Miroslaw Gilski ${ }^{2}$, Ivan Shabalin ${ }^{1}$, Marek Grabowski ${ }^{1}$, Bernhard Rupp ${ }^{6}$}

\author{
${ }^{1}$ University of Virginia, Charlottesville, United States of America; \\ ${ }^{2}$ A. Mickiewicz University, Poznan, Poland; \\ ${ }^{3}$ National Cancer Institute, United States of America; \\ ${ }^{4}$ University of Warsaw, Warsaw, Poland; \\ ${ }^{5}$ Poznan University of Technology, Poznan, Poland; \\ ${ }^{6} k$.-k Hofkristallamt, United States of America; \\ ${ }^{7}$ Polish Academy of Sciences, Poland \\ wladek@iwonka.med.virginia.edu
}

\begin{abstract}
Structural information, mainly derived by X-ray crystallography and Cryo-Electron Microscopy, is the quintessential prerequisite for structural-guided drug discovery. However, accurate structural information is only one piece of information necessary to understand the big picture of medical disorders. To provide a rapid response to emerging biomedical challenges and threats like COVID-19, we need to analyze medical data in the context of other in-vitro and in-vivo experimental results. Recent advancements in biochemical, spectroscopical, and bioinformatics methods may revolutionize drug discovery, albeit only when these data are combined and analyzed with effective data management framework like Advanced Information System proposed in 2017. The progress on AIS is too slow, but creating such a system is a Grand Challenge for biomedical sciences. By definition, a Grand Challenge is a challenging and extremely difficult long-term project that is not always appreciated by those looking for immediate returns.
\end{abstract}

Keywords: Covid-19, Advanced Information Systems, Structure validation 